\title{
Synchronization of Coupled Chaotic Circuits in Regular Tetrahedron Form
}

\author{
Takahiro Nagai, Yoko Uwate and Yoshifumi Nishio \\ Dept. of Electrical and Electronic Engineering, Tokushima University, \\ 2-1 Minamijosanjima, Tokushima, 770-8506 Japan \\ Email: \{nagataka, uwate, nishio\}@ee.tokushima-u.ac.jp
}

\begin{abstract}
This article presents synchronization phenomena of coupled chaotic circuits by regular tetrahedral form. We pay attentions to the behavior of each chaotic circuit and observe the synchronization states between adjacent circuits. In this circuit model, we can observe several patterns of synchronization phenomena.
\end{abstract}

\section{Introduction}

There are a lot of synchronization phenomena in natural environment. It is one of the nonlinear phenomena that we can actually observe. For example, swing of the pendulum, firefly luminescence, cardiac heartbeat etc. are well known as the synchronization phenomena. Synchronization phenomena have a feature that the set of small power can produce very big power by synchronizing at a time. Therefore, studies of synchronization phenomena have been widely reported not only engineering but also the physical and biological fields. Coupled chaotic circuits systems are suitable models to express many kinds of high-dimensional nonlinear phenomena. Especially, many researchers are attracted to chaotic mechanisms because chaotic synchronizations include complex behaviors [1]-[4].

In our basic investigations, we use several van der Pol oscillators. Van der Pol oscillators have been coupled in various form and have been investigated about their synchro-

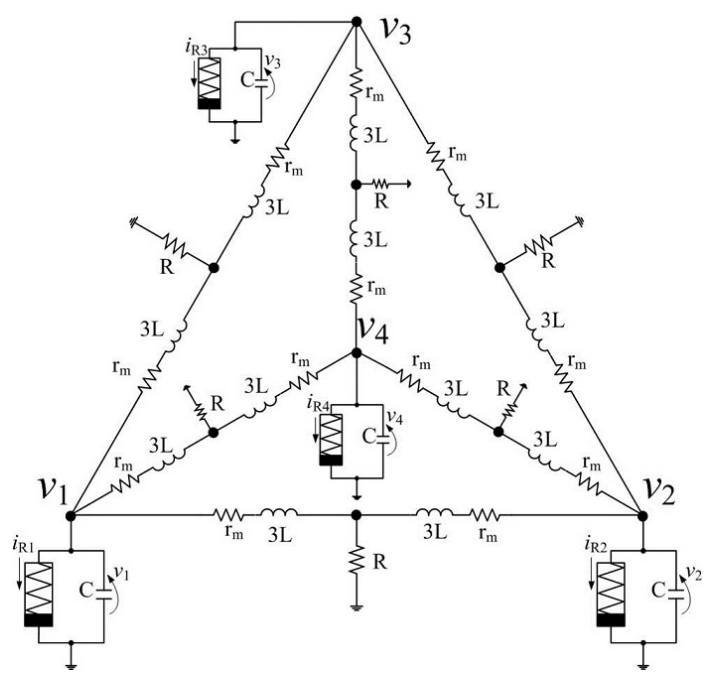

Figure 1: Four coupled oscillators.

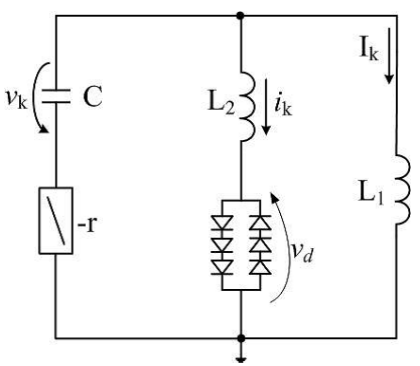

Figure 2: Chaotic circuit.

nization phenomena. Especially, we can observe very interesting synchronization phenomena when we couple each inductor and ground by coupling resistor $R$. For example, the circuit model which coupled three van der Pol oscillators as a ring topology has been found out the three phase synchronization (phase shift 120 degrees) by the interaction of each oscillator [5]. However, the three-phase synchronization was always observed stably in that system. In our previous study, we have investigated synchronization phenomena of coupled van der Pol oscillators in regular tetrahedral form (see Fig. 1) [6]. We could observe that the phase difference between adjacent oscillators changed and that the synchronization was destroyed after the adjacent oscillators synchronize with anti-phase. In other words, this circuit model has the feature of repeated anti-phase synchronous and asynchronous states.

In this study, we investigate synchronization phenomena in coupled chaotic circuits with tetrahedron form. We pay attentions to the behavior of each chaotic circuit and observe the synchronization states between adjacent circuits. Also, we make comparison between coupled oscillators and coupled chaotic oscillators and confirm the rotation of phase differences between adjacent chaotic circuits. Moreover, we research synchronization states by changing chaotic parameters.

\section{Circuit Model}

We show the chaotic circuits using in this investigation in Fig. 2. This circuit is composed of two types of inductors, a capacitor, a negative resistor and interactive diodes. We couple each chaotic circuit via $L_{1}$ and ground by coupling resistor $R$ in this study. We describe this circuit diagram of coupled chaotic circuits in Fig. 3. In the computer 


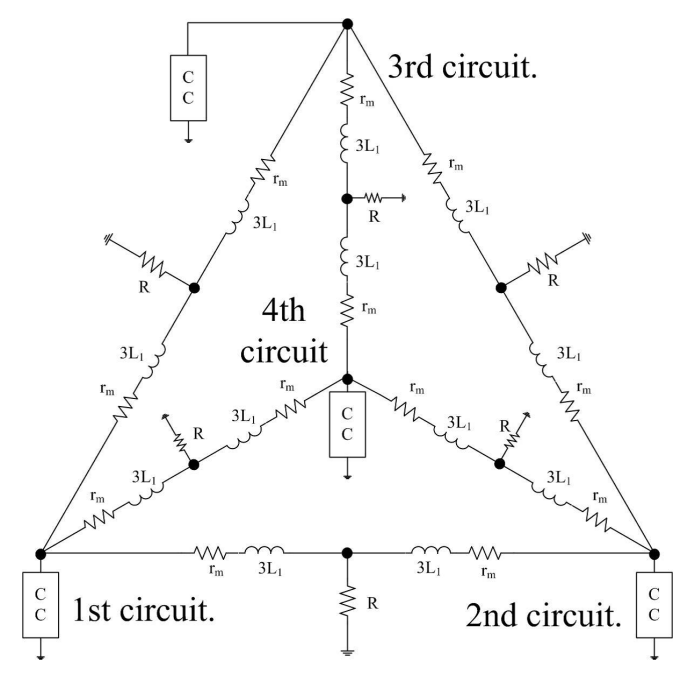

Figure 3: Circuit model for tetrahedron form.

simulations, we assume that the $v_{d}-i_{k}$ characteristics of the nonlinear resistor consisting of diodes by the following function.

$$
v_{d}\left(i_{k}\right)=\sqrt[9]{r_{d} i_{k}}
$$

The normalized circuit equations are expressed as:

$$
\left\{\begin{aligned}
\frac{d x_{a k}}{d \tau} & =\frac{1}{3}\left\{\left(x_{a k}+x_{b k}+x_{c k}+y_{k}\right)-z_{k}-\eta x_{a k}-\gamma\left(x_{a k}+x_{n}\right)\right\} \\
\frac{d x_{b k}}{d \tau} & =\frac{1}{3}\left\{\left(x_{a k}+x_{b k}+x_{c k}+y_{k}\right)-z_{k}-\eta x_{b k}-\gamma\left(x_{b k}+x_{n}\right)\right\} \\
\frac{d x_{c k}}{d \tau} & =\frac{1}{3}\left\{\left(x_{a k}+x_{b k}+x_{c k}+y_{k}\right)-z_{k}-\eta x_{c k}-\gamma\left(x_{c k}+x_{n}\right)\right\} \\
\frac{d y_{k}}{d \tau} & =\alpha \beta\left(x_{a k}+x_{b k}+x_{c k}+y_{k}\right)-z_{k}-f\left(y_{k}\right) \\
\frac{d z_{k}}{d \tau} & =\left(1+\Delta \omega_{k}\right)\left(x_{a k}+x_{b k}+x_{c k}+y_{k}\right) .
\end{aligned}\right.
$$

where

$$
\begin{gathered}
I_{k}=a \sqrt{\frac{C}{L_{1}}} x_{k}, i_{a k}=a \sqrt{\frac{C}{L_{1}}} y_{a k}, i_{b k}=a \sqrt{\frac{C}{L_{1}}} y_{b k}, \\
i_{c k}=a \sqrt{\frac{C}{L_{1}}} y_{c k}, v_{k}=a z_{k}, t=\sqrt{L_{1} C} \tau, \\
\alpha=\frac{L_{1}}{L_{2}}, \beta=r \sqrt{\frac{C}{L_{1}}}, \gamma=R \sqrt{\frac{C}{L_{1}}} \\
\eta=r_{m} \sqrt{\frac{C}{L}}, a=\sqrt[8]{r_{d} \sqrt{\frac{C}{L_{1}}}}
\end{gathered}
$$$$
(\mathrm{k}=1,2,3,4),
$$

and

$$
f\left(y_{k}\right)=\sqrt[9]{y_{k}} .
$$

$\beta$ is bifurcation parameter, $\gamma$ is coupling strength, $\eta$ indicates the resistive component of the inductor and $y_{n}$ denotes the current of adjacent oscillator. For computer simulations, in order to consider the difference of real circuit

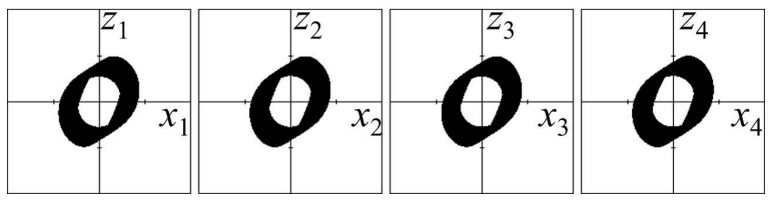

Figure 4: Attractor of each chaotic circuit (horizontal axis: $x_{k}$, vertical axis: $y_{k}(k=1,2,3,4)$.

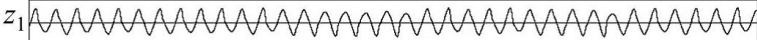

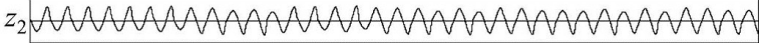 \\ $z_{3}$ WWNANNWNANMNNWWNANWNAN

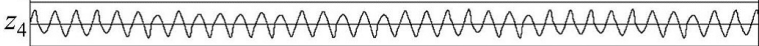

Figure 5: Time waveform of the each circuit.

elements, we suppose the frequency error $\Delta \omega_{k}$ and calculate the phase differences between adjacent chaotic circuits.

\section{Synchronization Phenomena}

We calculate Eq. (2) using the fourth-order Runge-Kutta method with the step size $h=0.001$. In this simulation, we fix the parameters $\alpha=20.0, \eta=0.00001, \Delta \omega_{1}=0.0, \Delta \omega_{2}$ $=0.001, \Delta \omega_{3}=0.002, \Delta \omega_{4}=0.003$, and change the bifurcation parameter or the coupling strength. As a result, we can find out very interesting phase synchronizations which could not been observed in four coupled oscillators. For example, we show the simulation result of the synchronization phenomena in Fig. 4 and Fig. 5. In Fig. 4, we show the attractor of each chaotic circuit and the horizontal axis is the electric current, and the vertical axis is the voltage. We set the parameters $\beta=0.330$ and $\gamma=0.250$. In this case, we can observe complex behaviors of time waveform and phase differences between adjacent chaotic circuits are shown as Fig. 6. In this figure, first and fourth circuit, second and third circuit synchronize in-phase and phase differences between adjacent other circuits are going to approach anti-phase. These synchronization states

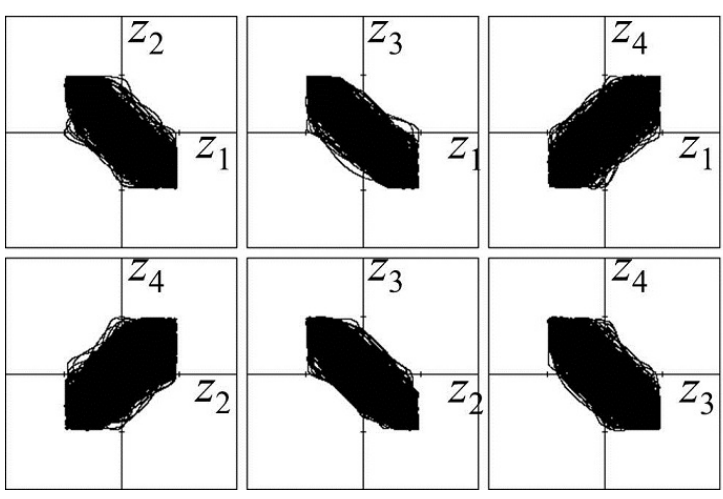

Figure 6: Lissajous figures. 


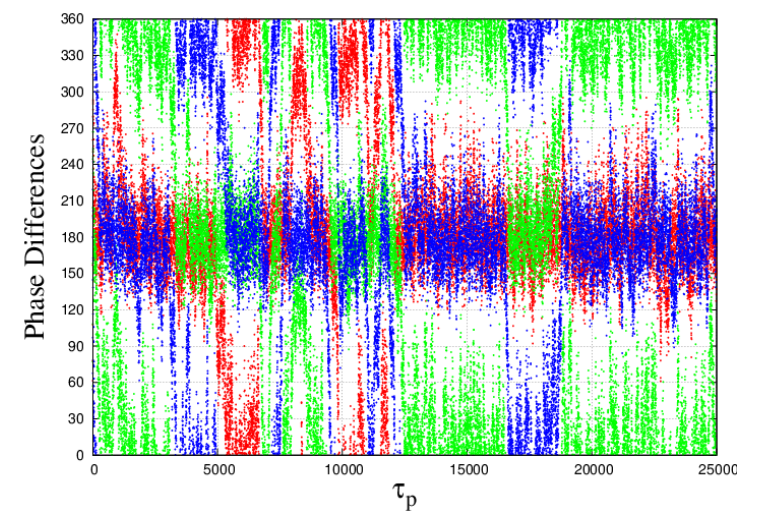

Figure 7: Phase shifts for $\beta=0.330$ and $\gamma=0.250$ (red is phase difference between 1st-2nd circuit, green is between $1 \mathrm{st}-3 \mathrm{rd}$, blue is between 1 st-4th).

keep changing in-phase and anti-phase by progress of time. Figure 7 shows the simulation result of long span which represented change of phase differences. Here we show that red dot indicates phase difference between 1 st and 2 nd circuit, green one is between 1st and 3rd, blue one is between 1 st and 4th. Even if initial conditions are changed, these synchronization phenomena do not have the qualitative change and synchronization states of beginning only change in-phase or anti-phase.

Next, we present the relationship of coupling strength and synchronization phenomena. We can see chaotic attractors in the case of week coupling strength and these change from chaos to periodic solution by becoming strong coupling strength. Change of attractors also affects synchronization phenomena between adjacent chaotic circuits. If the coupling strength become smaller than 0.200 , we can observe asynchronous between all of adjacent circuits.

Also, the four-phase synchronization appears when the coupling strength $\gamma=0.280$. At this time, phase differences synchronize in a particular area clearly as compared

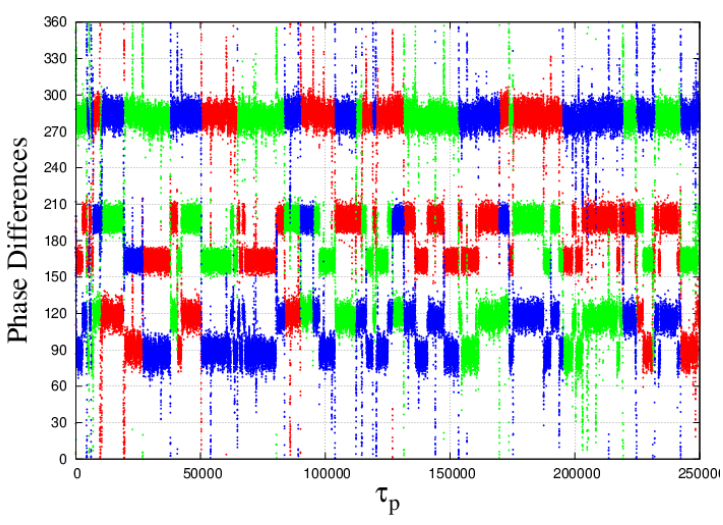

Figure 8: Phase shifts for $\beta=0.330$ and $\gamma=0.280$ (red is phase difference between 1st-2nd circuit, green is between $1 \mathrm{st}-3 \mathrm{rd}$, blue is between $1 \mathrm{st}-4 \mathrm{th})$.

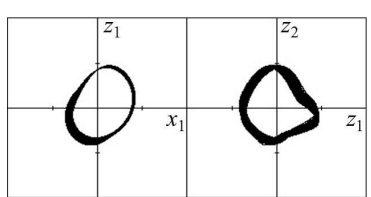

(a)

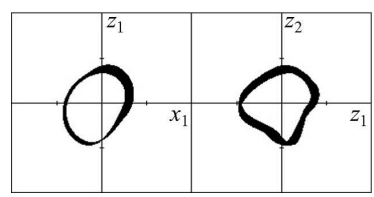

(c)

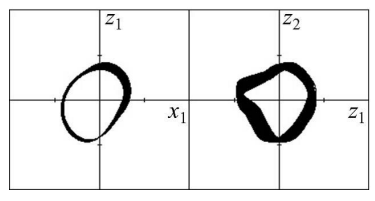

(e)

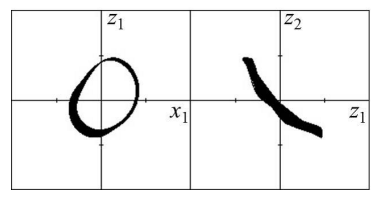

(b)

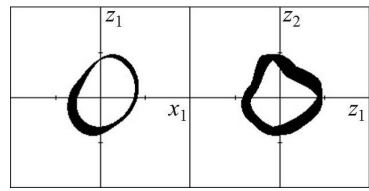

(d)

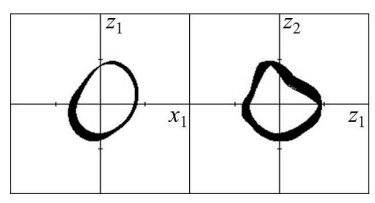

(f)
Figure 9: First attractor and phase shifts between 1st and 2nd circuits. (a) $\tau_{p}=3400$. (b) $\tau_{p}=14000$. (c) $\tau_{p}=$ 120000. (d) $\tau_{p}=142000$. (e) $\tau_{p}=182000$. (f) $\tau_{p}=$ 200000 .

to switching of in-phase and anti-phase. Figure 8 shows that synchronization states between first and the other circuits change by time. Namely, the red point shows the synchronization phenomena between first and second circuits. This means that two circuits synchronize 270 degrees first, after that phase difference shifts anti-phase and 90 degrees.

\section{Dependence on Bifurcation Parameter}

We can observe the phase shifts which are different from synchronization phenomena of former section by changing bifurcation parameter. In this section, we fix the coupling strength $\gamma=0.300$ and research the behaviors of synchronization states for each circuit. When $\beta$ is same value and the coupling strength differs, the chaotic attractors do not occur but the periodic attractors occur. For example, one-periodic attractor can be described when the parameter is $\beta=0.280$. Synchronization phenomena between adjacent circuits can be distinguished one anti-phase synchronization and two asynchronous. Also, we can observe switching of three different phase shift when $\beta=0.290$ (see Fig. 10(a)). This means that red point describes phase shift from 180 to 330 degrees, green one is from 30 to 180 degrees and blue one is switching of anti-phase and asynchronous. The other case, we can find out the phase shift which occurs alternately anti-phase synchronization and asynchronous in $\beta=0.305$ (see Fig. 10(b)). After that, four-phase synchronization occurs (see Fig. 10(c)).

Next, the chaotic parameter is over $\beta=0.335$, the attractors shift from one-periodic solution to two-periodic solution. Therefore we can see two types of phase differences over one synchronization phenomena between adjacent circuits. These phase differences appear alternately and keep 
Table 1: Change of synchronization states by $\beta$.

\begin{tabular}{|c|c|}
\hline$\beta$ & Synchronization States \\
\hline \hline$\sim 0.289$ & Anti-phase and asynchronous \\
\hline $0.289 \sim 0.300$ & Switching of different phase shift \\
\hline $0.300 \sim 0.305$ & Anti-phase and asynchronous \\
\hline $0.305 \sim 0.316$ & Switching of anti and asynchronous \\
\hline $0.316 \sim 0.335$ & Four-phase synchronization \\
\hline $0.335 \sim 0.336$ & Phase synchronization \\
\hline $0.336 \sim 0.345$ & Switching of in-phase and anti-phase \\
\hline $0.345 \sim$ & Divergence \\
\hline
\end{tabular}

synchronizing while maintaining the states. Also, asynchronous states are observed in whole chaotic networks before two-periodic solution appear. The time which shifts from asynchronous until periodic solution depends on the initial conditions. Two-periodic solution can be seen until $\beta$ $=0.336$, the attractors change chaos (see Fig. 10(d)). Then, we can see week switching of in-phase and anti-phase synchronization without phase synchronization.

\section{Conclusion}

This article presents synchronization phenomena which coupled chaotic circuits by regular tetrahedral form. In this circuit model, we have been able to observe several patterns synchronization phenomena. The observed switching in the former study about four coupled van der Pol oscillators sojourned only anti-phase area but we could confirm sojourn of in-phase area in this coupled chaotic circuits. Also, the four-phase synchronization are observed newly and we have found the feather which four-phase synchronization keeps shifting by 90 degrees depending on the bifurcation parameter. In the whole of coupled circuits, if bifurcation parameter is too strong or coupled strength is too week, this system is always asynchronous.

\section{Acknowledgments}

This work was partly supported by JSPS Grant-in-Aid for Young Scientists 23700269.

\section{References}

[1] L.M. Pecora and T.L. Carroll, "Synchronization in Chaotic Systems," Phys. Rev. Lett., vol.64, no.4, pp.821-824, 1990.

[2] Y. Nishio, K. Suzuki, S. Mori and A. Ushida, "Synchronization in Mutually Coupled Chaotic Circuits," Proc. of ECCTD'93, vol. 1, pp 637-642, Aug. 1993.

[3] M. Wada, Y. Nishio and A. Ushida,"Analysis of Bifurcation Phenomena on Two Chaotic Circuits Coupled by an Inductor" IEICE Trans. Fundamentals, vol.E80-A, no.5 , pp.869-875, May. 1997.

[4] H. Sekiya, S. Moro, S. Mori and I. Sasase "Synchronization Phenomena on Four Simple Chaotic Oscillators Full-Coupled by Capacitors" IEICE Trans. vol.J62-A, no.3 , pp.375-385, Mar. 1999.
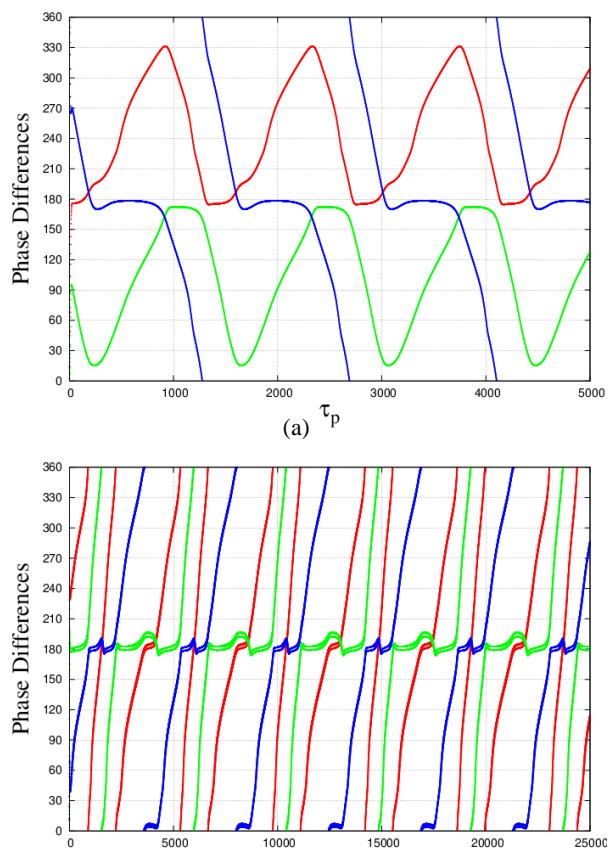

(b) $\tau^{2}$

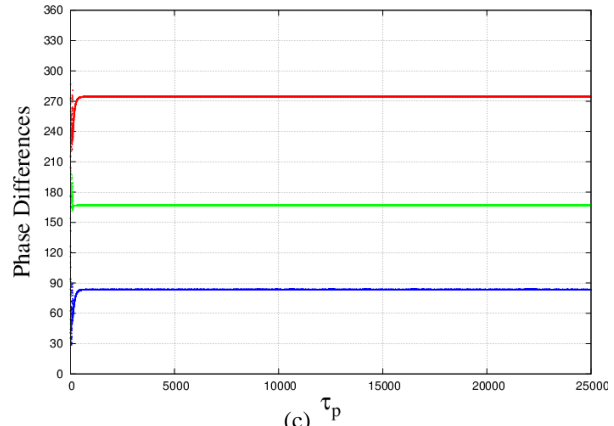

(c) ${ }^{\tau}$

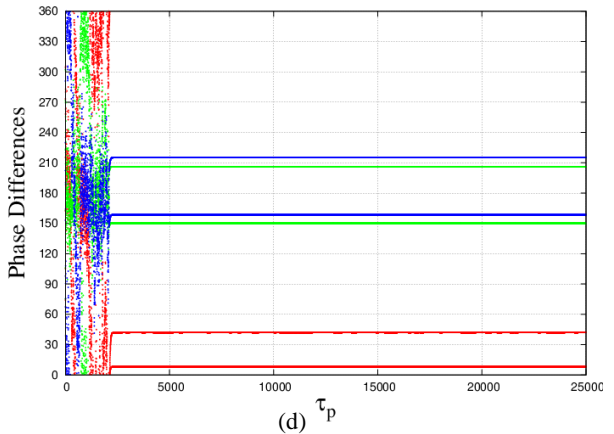

Figure 10: The phase shifts (red is phase difference between 1st-2nd circuit, green is between 1st-3rd, blue is between 1 st-4th). (a) $\beta=0.295$. (b) $\beta=0.305$. (c) $\beta=$ 0.330. (d) $\beta=0.336$.

[5] Y. Uwate, Y. Nishio and R Stoop, "Synchronization in Three Coupled van der Pol Oscillators with Different Coupling Strength," Proc. of NCSP'10, pp.109-112, Mar. 2010.

[6] T. Nagai, Y. Uwate and Y. Nishio, "Rotation of Phase Difference in Four Coupled Oscillators as a Regular Tetrahedron Form," Proc. of ECCTD'11, pp.762-765, Aug. 2011. 